\title{
Coherent interactions and long term evolution of ultrafast transients in a semiconductor laser
}

\author{
C. O’Rourke, J. Allam, K. Boehringer, A. Klaedtke, J. Hamm, O. Hess \\ Advanced Technology Institute, School of Electronics and Physical Sciences, \\ University of Surrey, Guildford, Surrey, GU2 $7 X H$, UK
}

\section{Introduction}

The interaction of short optical pulses with laser cavity modes is important in, for example, formation of mode-locked pulse trains, optical clock recovery, and external optical feedback [1]. In a dispersive nonlinear medium such as a semiconductor, a treatment of the optical field is required which goes beyond discrete modal frequencies. A description of coherent interactions requires resolution of the fast oscillating carrier wave on the femtosecond timescale, while the return to equilibrium may take place over nanoseconds due to a long cavity lifetime or relaxation oscillation period. In inhomogeneous structures such as multi-section or DFB lasers, sub-wavelength to millimeter spatial scales must also be described.

The spatio-temporal dynamics of the electric field may be calculated from Maxwell's equations using a finite-difference time-domain (FDTD) method. Coupling a Lorentzian resonance allows an approximate model of the optical gain [2]. Realistic microscopic models of the semiconductor gain based on the semiconductor Bloch equations [3] are at present too computationally intensive for simulation over such a range of temporal scales.

We consider a class of recent experiments in which a short optical pulse is injected into a semiconductor laser diode, allowing a study of the pulse-cavity interactions on time-scales shorter than the cavity roundtrip time. In addition to the expected pulse broadening and relaxation oscillations, new phenomena such as stable, long-lived 'dark pulses' were observed [4,5].

\section{Numerical Methods}

We have coupled an FDTD calculation of the electric field with multiple Lorentzian resonances which approximate the spectral dependence of the semiconductor gain. Figure 1 shows that four resonances provide a good fit to the semiconductor gain spectrum [6]. We found that spatially resolving the carrier lifetime and incorporating carrier diffusion was essential in achieving a physically realistic number of lasing modes.

\section{Results and discussion}

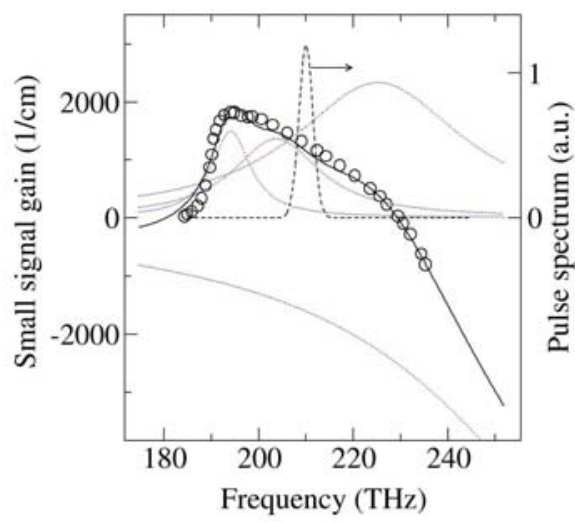

Figure 1: The solid line shows the resulting fit from the four Lorentzians (dotted lines). The circles are extracted from [6]. The dashed line shows the spectral width of a $180 \mathrm{fs}$ pulse.

During the propagation of a short pulse through a population-inverted semiconductor a region of depleted gain is left behind the injected pulse. For a laser under CW operation this region of depleted gain can evolve into a long lived 'dark pulse'. Figure 2 shows the simulated output from the laser facet following the injection of an optical pulse with a central frequency of $193 \mathrm{THz}$, resonant with the $\mathrm{CW}$ emission wavelength. The initial perturbation induces relaxation oscillations (period $\sim 50 \mathrm{ps)} \mathrm{which} \mathrm{persist} \mathrm{for} \sim 400 \mathrm{ps}$. A bright pulse is emitted from the facet every $\sim 7 \mathrm{ps,} \mathrm{after} \mathrm{each}$ cavity round-trip. The amplitude of successive bright pulses decays on the timescale of a few hundred ps. A dark pulse forms after the first few round trips, and is the dominant feature after $\sim 200$ ps. On a longer time scale, this feature oscillates (with $\sim 500$ ps period) between a predominantly bright and dark pulse superimposed on the CW emission. All of this behavior is in good qualitative agreement with experimental results [4]. Inset (A) of Figure 2 shows the CW spectrum calculated from the windowed Fourier transform of the emission $50 \mathrm{ps}$ prior to injection of the pulse. Inset (B)

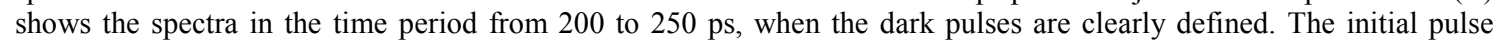

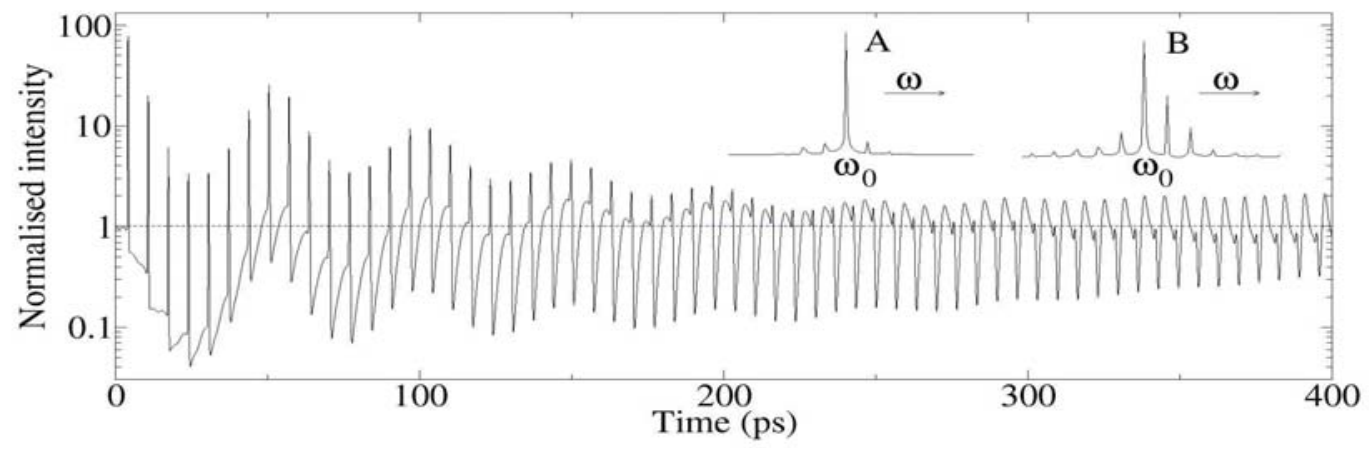

Figure 2: Pulse train following the injection of a 180 fs pulse into a $300 \mu \mathrm{m}$ long uncoated Fabry-Perot laser diode. The pulse train has been normalized to the $\mathrm{CW}$ intensity. 
injection has excited additional meta-stable longitudinal cavity modes which are responsible for the long-lived dark pulses.

We investigate the spectral dynamics by cross-correlating the electric field with a $1 \mathrm{ps}$ gating pulse to extract the frequency- and time-dependent intensity, analogous to a "FROG" experiment. Figure $3 \mathrm{~B}$ shows the $2^{\text {nd }}$ and $3^{\text {rd }}$ emitted pulses for resonant injection, the injected pulse having the same central frequency as the main lasing mode. Figure $3 \mathrm{~A}$ shows nonresonant injection. Figure $3 \mathrm{C}$ shows the pulse train after $\sim 250 \mathrm{ps}$ when the dark pulses are well-formed, and 3D shows their evolution into bright pulses at later times.

The interaction between the injected pulse and the standing waves present in the laser cavity is dependent on the relative phase of their carrier waves. When in antiphase, destructive interference occurs: the intensity 'holes' clearly visible in figures $3 \mathrm{~A}$ and $3 \mathrm{~B}$ arise from the destructive interference. For different values of the relative phase, the transients evolve differently. It is therefore essential to consider the role of the phase in dark pulse formation.

Typical experiments, in which the delay time of the gating pulse is varied slowly compared to the repetition rate of the pulse source, effectively average over many data sets with random relative phase. Additionally, in the absence of active stabilization of path and cavity lengths, the arrival time of the injected pulse also varies with respect to the 'self pulsations' due to interference between longitudinal modes involved in the $\mathrm{CW}$ emission. Hence Figure 2 is an average of 30 simulations with a range of carrier arrival times and phases.

Coherent interactions are strongest when the propagating transient and the standing waves modes have similar amplitude. When in antiphase, this leads to coherent destruction of the $\mathrm{CW}$ emission, gain enhancement, and reversal of the phase of relaxation oscillations. For all investigated pulse intensities, the propagating picosecond dark pulse and the CW laser emission have comparable amplitude once the bright pulse has decayed. We conclude that coherent interactions are the origin of the dark pulse / bright pulse oscillations shown in 3C and 3D, where a small difference in the group velocity for the propagating transient and the $\mathrm{CW}$ emission leads to alternating destructive and constructive interference. In contrast, earlier work employing single-mode traveling-wave rate equations [5] ascribed this behavior to variation of the carrier temperature.
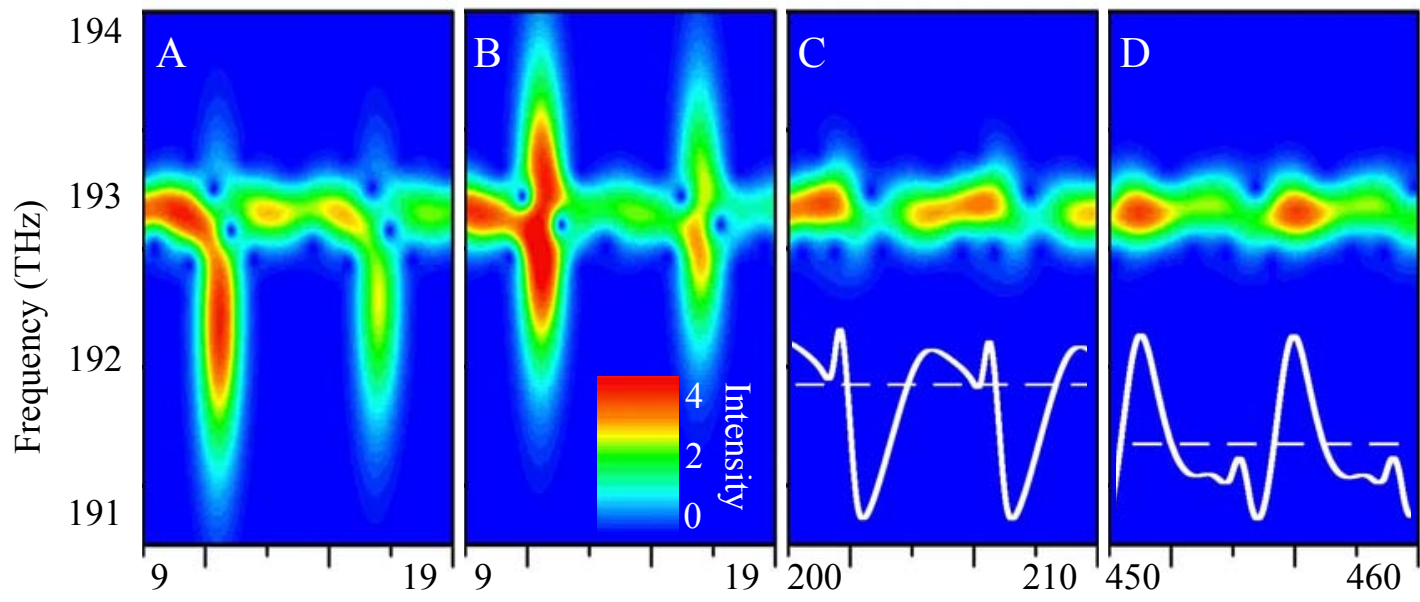

Time $(\mathrm{ps})$

Figure 3: Plots A and B show the injection of a non-resonant and resonant pulse, showing the second and third round trip. C and D show dark pulses persisting after $250 \mathrm{ps}$ and bright pulses after $450 \mathrm{ps}$ for the case of resonant injection. The insets show the electric field envelope relative to the $\mathrm{CW}$ level.

\section{Conclusion}

We have investigated the coherent interaction between standing waves of a $\mathrm{CW}$ laser and an optical pulse injected into the cavity, and the formation and subsequent evolution of 'dark pulses'. We have found that the persistence of the dark pulses and the dark-pulse bright-pulse oscillations can be attributed to coherent effects. Our simulation method is a useful tool for studying long term evolution of coherent effects in semiconductor laser cavities such as the generation of mode-locked pulse trains, optical clock recovery and external optical feedback.

Carl O'Rourke acknowledges financial support from the EPSRC

\section{References}

[1] P. Vasil'ev, Ultrafast Diode Lasers: Fundamentals and Applications, Artech House, 1995.

[2] A.S. Nagra, R.A. York, IEEE Trans. Antennas Propagat. 46 (1998) 334.

[3] W.W. Chow, S.W. Koch, Semiconductor-Laser Fundamentals: Physics of the Gain Materials, Springer, 1999.

[4] M. Kauer, J.R.A. Cleaver, J.J. Baumberg, A.P. Heberle, Appl. Phys. Lett. 72 (1998) 1626.

[5] M. Kauer, A.P. Heberle, J. Allam, J.J. Baumberg, J.R.A. Cleaver, Physica B 272 (1999) 394.

[6] M. Rosenzweig, M. Möhrle, H. Düser, H. Venghaus, IEEE J. Quantum Electron., 27 (1991) 1804 